\title{
Étude électrochimique des systèmes binaires et ternaire engageant les éléments bismuth, antimoine et tellure
}

\author{
D. Del Frari, S. Diliberto, C. Boulanger et J.M. Lecuire
}

Laboratoire d'Électrochimie des Matériaux, UMR CNRS 7555, Université de Metz, 1 Bd. Arago, Technopôle, CP 87811, 57078 Metz Cedex 3, France

\begin{abstract}
Résumé. Dans la présente étude, nous nous sommes intéressés à la recherche des meilleures conditions de synthèse du composé $\mathrm{Bi}_{0,5} \mathrm{Sb}_{1,5} \mathrm{Te}_{3}$, présentant des propriétés thermoélectriques optimales à température ambiante pour un composé de type $\mathrm{p}$. Une étude voltampérométrique a donc été menée pour les binaires engageant ces trois éléments, c'est-à-dire $\mathrm{Bi}$ - $\mathrm{Te}$ et $\mathrm{Sb}$-Te, ainsi que pour le ternaire $\mathrm{Bi}-\mathrm{Sb}-\mathrm{Te}$. Les ions $\mathrm{Bi}^{\mathrm{III+}}, \mathrm{Sb}^{\mathrm{III+}}$ et $\mathrm{Te}^{\mathrm{IV+}}$ ont été mis en solution dans l'électrolyte acide perchlorique $\mathrm{HClO}_{4} 1 \mathrm{M}-$ acide tartrique $\mathrm{C}_{4} \mathrm{H}_{6} \mathrm{O}_{6} 0,1 \mathrm{M}$, avec une concentration maximale en tellure de l'ordre de $10^{-2} \mathrm{M}$. Les rapports envisagés entre les ions, choisis en fonction d'études antérieures, sont les suivants : $\mathrm{Bi} / \mathrm{Te}=1$ et $\mathrm{Sb} / \mathrm{Te}=1$ pour les binaires, et $(\mathrm{Bi}+\mathrm{Sb}) / \mathrm{Te}=1$ et $\mathrm{Sb} / \mathrm{Bi}=3$ pour le ternaire. Des dépôts ont été réalisés à différents potentiels, de manière à identifier les pics obtenus sur les voltampérogrammes. Ces dépôts ont été caractérisés par diffraction des rayons $\mathrm{X}$, et leur stoechiométrie a été obtenue par microsonde de Castaing.
\end{abstract}

\section{INTRODUCTION}

La réalisation de matériaux thermoélectriques par électrodéposition présente un intérêt croissant pour certaines applications comme la miniaturisation de modules Peltier. Cette technique est simple de mise en œuvre et permet de contrôler très finement la composition des films de semi-conducteurs. Les matériaux principalement étudiés à température ambiante sont les semi-conducteurs suivants : $\mathrm{Bi}_{2-\mathrm{x}} \mathrm{Te}_{3}$ (type $\mathrm{n}$ ), $\mathrm{Bi}_{2} \mathrm{Te}_{3-\mathrm{y}}$ (type $\mathrm{p}$ ), et les ternaires plus performants $\mathrm{Bi}_{2}(\mathrm{Te}, \mathrm{Se})_{3}(\mathrm{n})$ et $(\mathrm{Bi}, \mathrm{Sb})_{2} \mathrm{Te}_{3}(\mathrm{p})[1]$. Dans la présente étude, nous nous sommes intéressés à la recherche des meilleures conditions de synthèse du composé $\mathrm{Bi}_{0,5} \mathrm{Sb}_{1,5} \mathrm{Te}_{3}$, présentant des propriétés thermoélectriques optimales.

Le mécanisme de formation de ce composé est basé sur la réduction des ions tellurite en présence de bismuth et d'antimoine, selon la réaction suivante :

$$
2(1-\mathrm{x}) \mathrm{Bi}^{3+}+2 \mathrm{x} \mathrm{Sb}^{3+}+3 \mathrm{Te}^{+\mathrm{IV}}+18 \mathrm{e}^{-} \rightarrow\left(\mathrm{Bi}_{1-\mathrm{x}} \mathrm{Sb}_{\mathrm{x}}\right)_{2} \mathrm{Te}_{3}
$$

Cette étude est consacrée à l'analyse voltampérométrique des systèmes $\mathrm{Bi}-\mathrm{Te}$, $\mathrm{Sb}$-Te et $\mathrm{Bi}-\mathrm{Sb}-\mathrm{Te}$.

\section{PARTIE EXPERIMENTALE}

Les courbes voltampérométriques ont été réalisées en utilisant un montage à 3 électrodes, à $25^{\circ} \mathrm{C}$. L'électrode de travail est un disque de platine ( $2 \mathrm{~mm}$ de diamètre). L'électrode de référence est une électrode au calomel à $\mathrm{KCl}$ saturé, et l'électrode auxiliaire est constituée d'un disque de platine (10 $\mathrm{mm}$ de diamètre). Les dépôts ont été réalisés sur inox poli au diamant $(1 \mu \mathrm{m})$, de surface $2 \mathrm{~cm}^{2}$. 
Le dispositif engage un potentiostat Radiometer PGZ 301, relié à un micro-ordinateur piloté par le logiciel Voltamaster 4. L'étude voltampérométrique et les dépôts ont été réalisés en régime de diffusion naturelle, à une vitesse de balayage très lente $(\mathrm{v}=0,5 \mathrm{mV} / \mathrm{s})$.

Pour assurer la stabilité et la solubilité des espèces, les ions $\mathrm{Bi}^{\mathrm{III}+}, \mathrm{Sb}^{\mathrm{III+}}$ et $\mathrm{Te}^{\mathrm{IV}+}$ ont été mis en solution dans l'électrolyte acide perchlorique $\mathrm{HClO}_{4} 1 \mathrm{M}$ - acide tartrique $\mathrm{C}_{4} \mathrm{H}_{6} \mathrm{O}_{6} 0,1 \mathrm{M}$. Les solutions ont respectivement été obtenues par dissolution des oxydes $\mathrm{Bi}_{2} \mathrm{O}_{3}, \mathrm{Sb}_{2} \mathrm{O}_{3}$ et $\mathrm{TeO}_{2}$. La concentration en tellure est constante et a été fixée à $10^{-2} \mathrm{M}$ pour toutes les solutions.

Les dépôts ont été caractérisés par diffraction des rayons X (INEL XRG 2500, à détecteur courbe CPS 120) et leurs stoechiométries ont été obtenues par microsonde de Castaing (CAMECA SX50 et SX100).

\section{RÉSULTATS ET DISCUSSION}

\section{1 Étude des solutions contenant les ions $\mathrm{Bi}^{\mathrm{III}+}$ et $\mathrm{Te}^{\mathrm{IV}+}$}

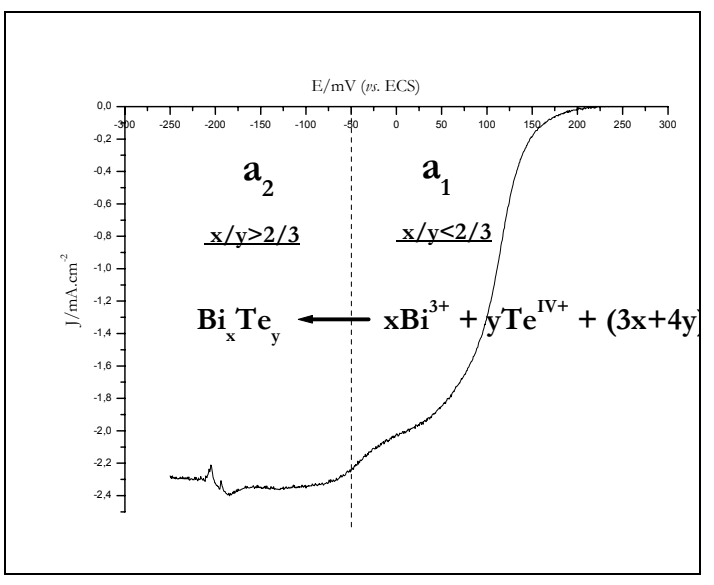

Figure 1. Système Bi- $\mathrm{Te}-[\mathrm{Bi}]=[\mathrm{Te}]=10^{-2} \mathrm{M}$ $\left(1 \mathrm{M} \mathrm{HClO}_{4}-0,1 \mathrm{M} \mathrm{C} \mathrm{C}_{4} \mathrm{H}_{6} \mathrm{O}_{6}\right.$ ) - Vitesse de balayage : $0,5 \mathrm{mV} / \mathrm{s}$.

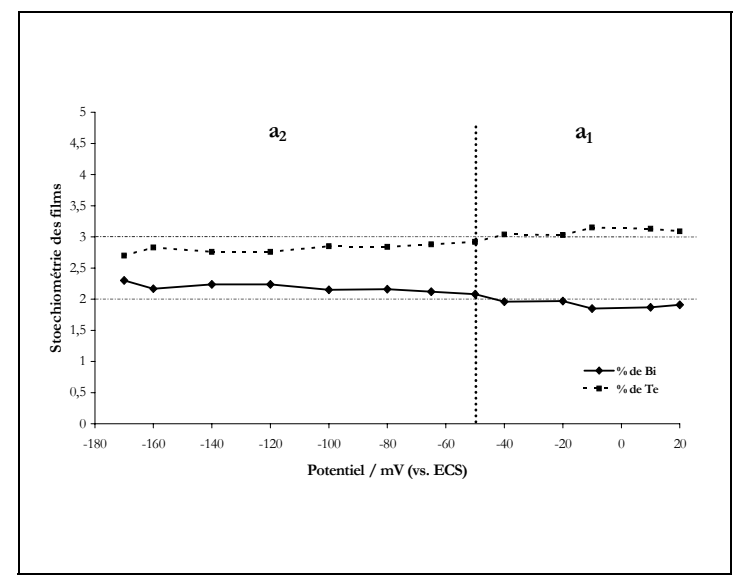

Figure 2. Variation de la stoechiométrie de $\mathrm{Bi}_{\mathrm{x}} \mathrm{Te}_{\mathrm{y}}$ en fonction du potentiel de dépôt $-[\mathrm{Bi}]=[\mathrm{Te}]=10^{-2} \mathrm{M}$.

Dans le but d'obtenir $\mathrm{Bi}_{2} \mathrm{Te}_{3}$, le rapport $[\mathrm{Bi}] /[\mathrm{Te}]$ a été fixé à 1. Ce rapport a été choisi en fonction d'études antérieures menées sur le tellurure de bismuth en milieu nitrique 1M [2-6].

La courbe cathodique retour de cette étude voltampérométrique présente deux paliers de réduction (Figure 1). Le premier $\left(a_{1}\right)$ est compris entre 50 et $-50 \mathrm{mV} / \mathrm{SCE}$. Le second $\left(\mathrm{a}_{2}\right)$ est situé à des potentiels plus cathodiques, allant de -50 à $-250 \mathrm{mV} / \mathrm{ECS}$. Au-delà se trouve le mur de réduction du solvant.

Les analyses stoechiométriques et cristallographiques ont été effectuées à différents potentiels et sont observables sur les Figures 2 et 3. Pour des potentiels relatifs au premier palier $\left(a_{1}\right)$, les dépôts ont une structure de type $\mathrm{Bi}_{2} \mathrm{Te}_{3}$, et une composition présentant un léger excès de tellure pour des potentiels plus anodiques. Concernant le second palier $\left(\mathrm{a}_{2}\right)$, les dépôts présentent toujours une structure de type $\mathrm{Bi}_{2} \mathrm{Te}_{3}$ mais avec un excès de bismuth. En outre, les diffractogrammes mettent en évidence la présence d'une orientation préférentielle suivant le plan (1.1.0) lorsque le dépôt s'enrichit en tellure. 


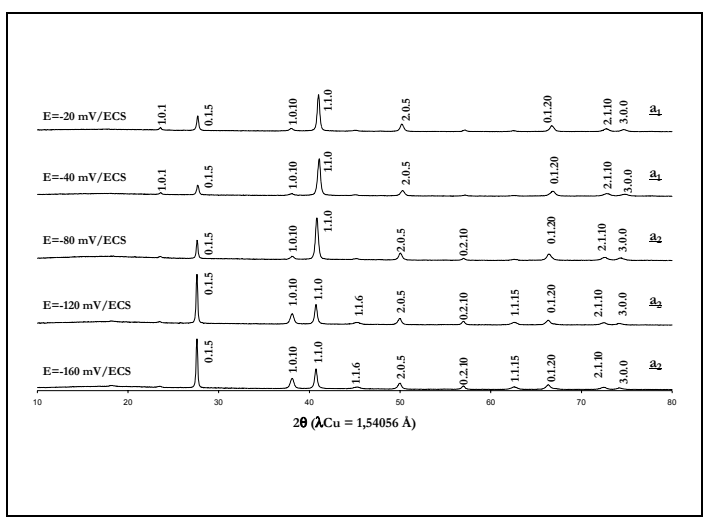

Figure 3. Diffraction des rayons $\mathrm{X}$ - Système Bi-Te - $[\mathrm{Bi}]=[\mathrm{Te}]=10^{-2} \mathrm{M}$.

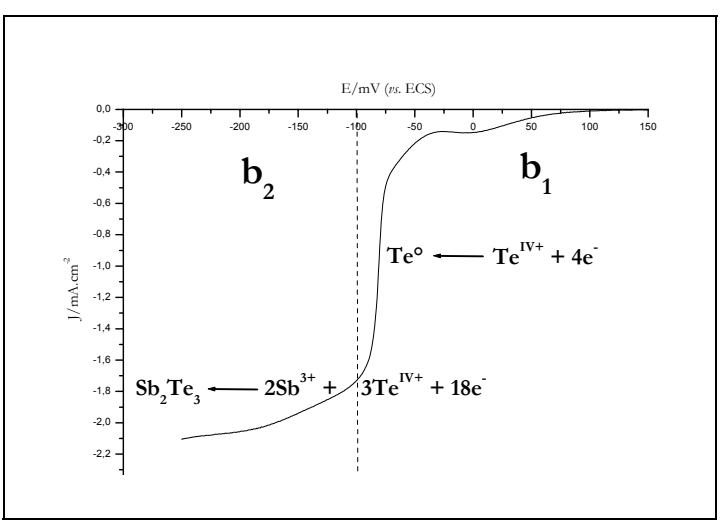

Figure 4. Système $\mathrm{Sb}-\mathrm{Te}-[\mathrm{Bi}]=[\mathrm{Te}]=10^{-2} \mathrm{M} \quad(1 \mathrm{M}$ $\mathrm{HClO}_{4}-0,1 \mathrm{M} \mathrm{C}_{4} \mathrm{H}_{6} \mathrm{O}_{6}$, $)$-Vitesse de balayage: $0,5 \mathrm{mV} / \mathrm{s}$.

\section{2 Étude des solutions contenant les ions $\mathrm{Sb}^{\mathrm{III}+}$ et $\mathrm{Te}^{\mathrm{IV}+}$}

Le mécanisme de formation de $\mathrm{Sb}_{2} \mathrm{Te}_{3}$ étant supposé équivalent à celui de $\mathrm{Bi}_{2} \mathrm{Te}_{3}$, les proportions entre $[\mathrm{Sb}]$ et $[\mathrm{Te}]$ sont identiques à celles des solutions de Bi-Te. Ainsi, $[\mathrm{Sb}] /[\mathrm{Te}]=1$ et $[\mathrm{Te}]=10^{-2} \mathrm{M}$. $\mathrm{La}$ courbe cathodique retour présente deux paliers de réduction (Figure 4$)$. Le premier $\left(\mathrm{b}_{1}\right)$, compris entre 50 et $-50 \mathrm{mV} / \mathrm{ECS}$, est représentatif de la réduction des ions $\mathrm{HTeO}_{2}{ }^{+}$en tellure métal. Le second $\left(b_{2}\right)$, situé à des potentiels plus cathodiques concerne la formation du binaire $\mathrm{Sb}_{2} \mathrm{Te}_{3}$. Une étude potentiostatique menée pour ces gammes de potentiel est venue confirmer ces conclusions (Figure 5). Ainsi, pour des potentiels cathodiques allant de -110 à $-250 \mathrm{mV} / \mathrm{ECS}$, les dépôts obtenus ont une stoechiométrie proche du tellurure d'antimoine $\mathrm{Sb}_{2} \mathrm{Te}_{3}$. Au-dessus de $-110 \mathrm{mV} / \mathrm{ECS}\left(\mathrm{b}_{1}\right)$, la stoechiométrie évolue brusquement vers des films composés majoritairement de tellure.

Une étude structurale a été menée sur un dépôt obtenu par voie potentiostatique à $-170 \mathrm{mV} / \mathrm{ECS}$ et présentant une stoechiométrie proche de $\mathrm{Sb}_{2} \mathrm{Te}_{3}$. Ce composé étant amorphe à température ambiante (Figure 6), sa cristallisation a été suivie par diffraction des rayons $\mathrm{X}$ à l'aide d'un diffractomètre Philipps X Pert Pro, équipé d'un four sous vide secondaire. Les clichés de diffraction ont été effectués tout les $100^{\circ} \mathrm{C}$ jusqu'à $400^{\circ} \mathrm{C}$. Ces clichés montrent une cristallisation du film en $\mathrm{Sb}_{2} \mathrm{Te}_{3}$ dès $100^{\circ} \mathrm{C}$. Les spectres obtenus ont été indexés à partir de la fiche ASTM 15-874 de $\mathrm{Sb}_{2} \mathrm{Te}_{3}$.

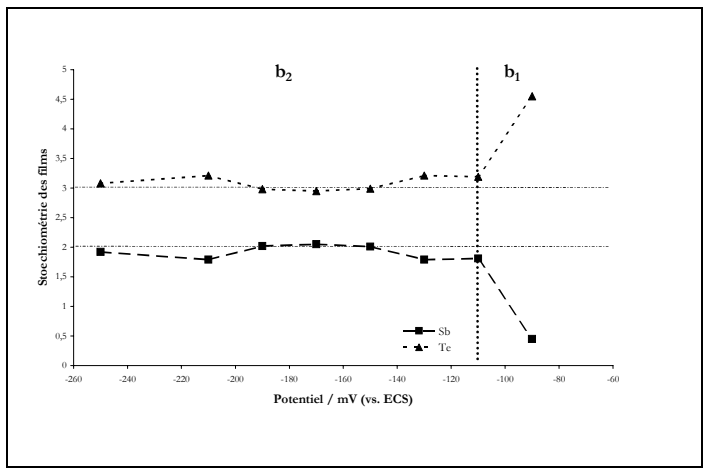

Figure 5. Variation de la stoechiométrie de $\mathrm{Sb}_{\mathrm{x}} \mathrm{Te}_{\mathrm{y}}$ en fonction du potentiel de dépôt $-[\mathrm{Bi}]=[\mathrm{Te}]=$ $10^{-2} \mathrm{M}$.

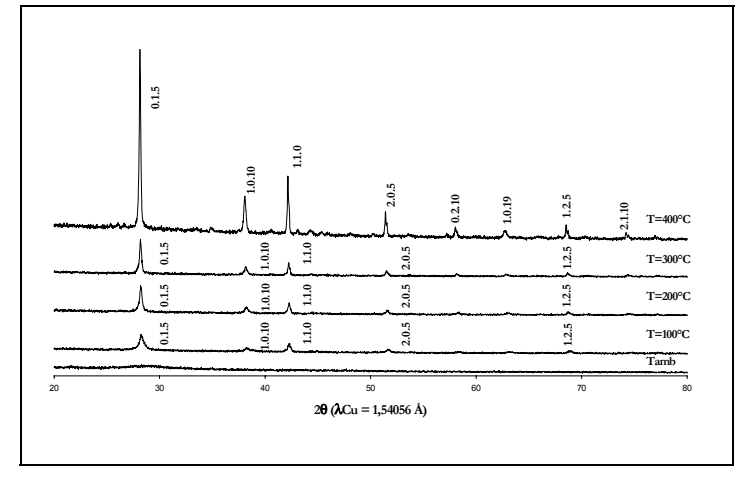

Figure 6. Suivi des traitements thermiques par DRX - Système Sb-Te - $[\mathrm{Sb}]=[\mathrm{Te}]=10^{-2} \mathrm{M}-\mathrm{E}_{\text {déposition }}=$ $-170 \mathrm{mV} / \mathrm{ECS}$. 


\section{3 Étude des solutions contenant les ions $\mathrm{Bi}^{\mathrm{III}+}, \mathrm{Sb}^{\mathrm{III+}}$ et $\mathrm{Te}^{\mathrm{IV+}}$}

\subsubsection{Influence du potentiel}

L'obtention des binaires $\mathrm{Sb}_{2} \mathrm{Te}_{3}$ et $\mathrm{Bi}_{2} \mathrm{Te}_{3}$ en milieu tartrique - perchlorique nous a permis de déterminer les conditions opératoires optimales visant à élaborer le ternaire $\mathrm{Bi}_{0,5} \mathrm{Sb}_{1,5} \mathrm{Te}_{3}$. C'est pourquoi la solution initiale du ternaire garde le même rapport de 1 entre les ions $(\mathrm{Bi}+\mathrm{Sb})$ et l'ion $\mathrm{Te}$. Concernant le rapport $\mathrm{Sb} / \mathrm{Bi}$, la stoechiométrie du ternaire souhaité, c'est-à-dire $\mathrm{Bi}_{0,5} \mathrm{Sb}_{1,5} \mathrm{Te}_{3}$, a gouverné notre choix, et nous l'avons fixé à $\mathrm{Sb} / \mathrm{Bi}=3$.

Une étude voltampérométrique a été réalisée, et la courbe cathodique retour présente trois paliers de réduction (Figure 7). Le premier palier $\left(\mathrm{c}_{1}\right)$, compris entre -50 et $50 \mathrm{mV} / \mathrm{ECS}$, peut tout aussi bien être représentatif de la réduction de $\mathrm{Te}^{\mathrm{IV}+}$ en tellure métal que de l'obtention de $\mathrm{Bi}_{2} \mathrm{Te}_{3}$ sur la base des voltampérogrammes précédents. Le troisième palier $\left(\mathrm{c}_{3}\right)$, situé entre -250 et $-150 \mathrm{mV} / \mathrm{ECS}$, est caractéristique de l'obtention du ternaire $\mathrm{Bi}_{0,5} \mathrm{Sb}_{1,5} \mathrm{Te}_{3}$. Le palier intermédiaire $\left(\mathrm{c}_{2}\right)$ concerne l'obtention d'un ternaire de type $\mathrm{Bi}_{\mathrm{x}} \mathrm{Sb}_{\mathrm{y}} \mathrm{Te}_{\mathrm{z}}$.

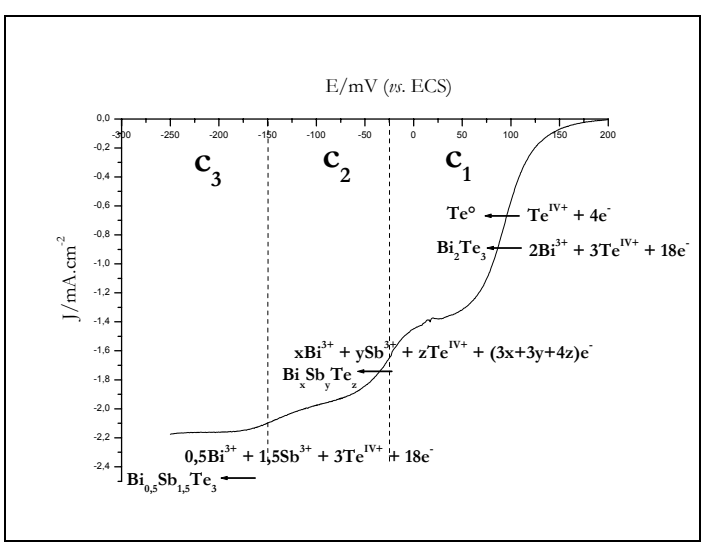

Figure 7. Système $\mathrm{Bi}-\mathrm{Sb}-\mathrm{Te}-([\mathrm{Bi}]+[\mathrm{Sb}]) /[\mathrm{Te}]=1-$ $[\mathrm{Sb}] /[\mathrm{Bi}]=3-[\mathrm{Te}]=10^{-2} \mathrm{M} \quad\left(1 \mathrm{M} \quad \mathrm{HClO}_{4^{-}}\right.$ $0,1 \mathrm{M} \mathrm{C}_{4} \mathrm{H}_{6} \mathrm{O}_{6}$, -Vitesse de balayage: $0,5 \mathrm{mV} / \mathrm{s}$.

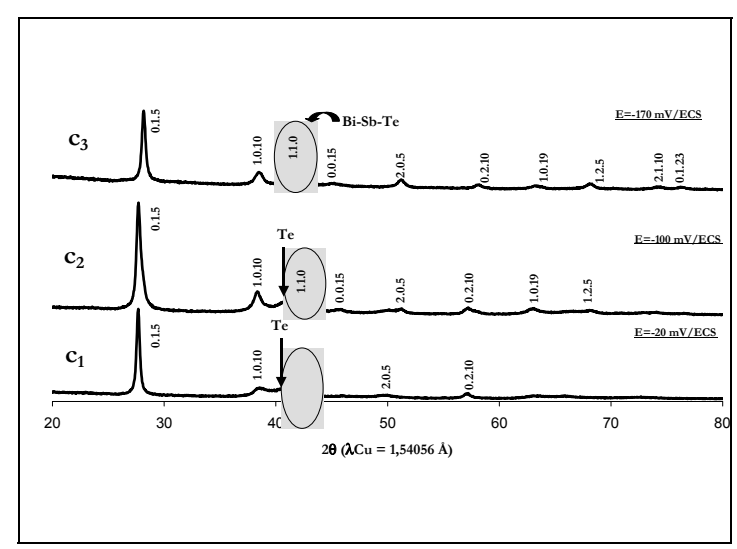

Figure 8. Diffraction des rayons $\mathrm{X}-$ Système $\mathrm{Bi}-\mathrm{Sb}-\mathrm{Te}-([\mathrm{Bi}]+[\mathrm{Sb}]) /[\mathrm{Te}]=1-[\mathrm{Sb}] /[\mathrm{Bi}]=3 \quad-$ $[\mathrm{Te}]=10^{-2} \mathrm{M}$.

Une étude cristallographique a été menée pour des dépôts réalisés sur ces trois paliers et les résultats sont présentés sur la Figure 8 . Ainsi, pour le palier situé à des potentiels très cathodiques $\left(\mathrm{c}_{3}\right)$, le spectre obtenu à un potentiel de $-170 \mathrm{mV} / \mathrm{ECS}$ présente la raie caractéristique de $\mathrm{Bi}_{0,5} \mathrm{Sb}_{1,5} \mathrm{Te}_{3}(11.0)$, et tous les pics ont été indexés à partir de la fiche ASTM 49-1713 de ce ternaire. Le dépôt obtenu est cristallisé et monophasé. Pour des potentiels moins cathodiques, représentatifs du second palier $\left(\mathrm{c}_{2}\right)$, un dépôt a été effectué à $-100 \mathrm{mV} / \mathrm{ECS}$. Le spectre obtenu présente une structure de type $\mathrm{Bi}_{0,5} \mathrm{Sb}_{1,5} \mathrm{Te}_{3}$, justifié par sa raie caractéristique, avec un léger décalage des pics vers la gauche du à un enrichissement en bismuth. En outre, une raie propre au tellure métal est apparue. Le palier situé entre -50 et $50 \mathrm{mV} / \mathrm{ECS}\left(\mathrm{c}_{1}\right)$ a enfin été étudié pour un potentiel de dépôt de $-20 \mathrm{mV} / \mathrm{ECS}$. Le cliché obtenu ne présente pas la raie caractéristique du ternaire, mais une structure de type $\mathrm{Bi}_{2} \mathrm{Te}_{3}$ avec présence de la raie de tellure métal. Ces résultats se justifient par le fait que l'antimoine ne se réduit pas aux potentiels du palier $\left(\mathrm{c}_{1}\right)$.

Les résultats stoechiométriques sont observables sur la Figure 9. Pour des potentiels correspondant au premier palier $\left(c_{1}\right)$, les dépôts sont composés essentiellement de tellure et de bismuth, devant se 
trouver sous la forme de $\mathrm{Bi}_{2} \mathrm{Te}_{3}$ et de $\mathrm{Te}^{\circ}$ d'après l'étude cristallographique. Ces deux phases ne sont pas différenciables par microsonde de Castaing. Concernant les potentiels du second palier $\left(\mathrm{c}_{2}\right)$, les résultats stoechiométriques confirment l'obtention d'un ternaire de type $\mathrm{Bi}_{\mathrm{x}} \mathrm{Sb}_{\mathrm{y}} \mathrm{Te}_{\mathrm{z}}$, avec un excès de tellure, ce qui confirme les études structurales (Figure 8). Les courbes montrent que la composition est fortement dépendante du potentiel. En effet, le tellure et l'antimoine évoluent de façon antagoniste, alors que la courbe de Bi reste constante à 0,5 . Enfin, pour le troisième palier $\left(\mathrm{c}_{3}\right)$, la composition du film de $\mathrm{Bi}_{0,5} \mathrm{Sb}_{1,5} \mathrm{Te}_{3}$ est indépendante du potentiel pour une gamme de -300 à $-150 \mathrm{mV} / \mathrm{ECS}$. Ces résultats viennent donc corroborer les études cristallographiques et voltampérométriques menées précédemment.

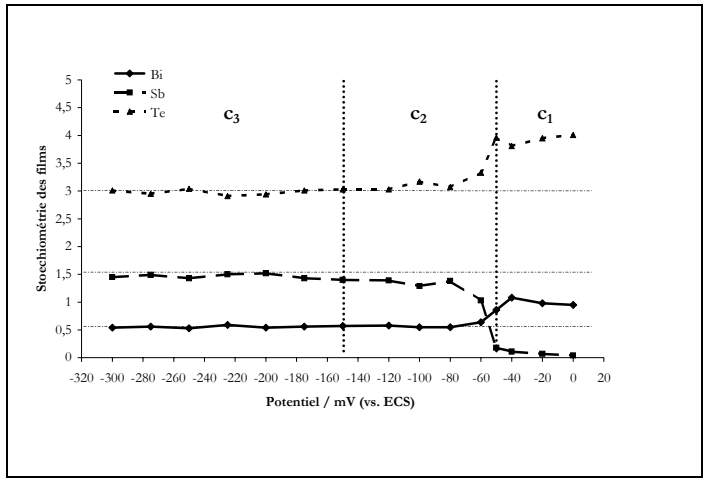

Figure 9. Variation de la stoechiométrie de $\mathrm{Bi}_{\mathrm{x}} \mathrm{Sb}_{\mathrm{y}} \mathrm{Te}_{\mathrm{z}}$ en fonction du potentiel de dépôt $([\mathrm{Bi}]+[\mathrm{Sb}]) /[\mathrm{Te}]=1-[\mathrm{Sb}] /[\mathrm{Bi}]=3-[\mathrm{Te}]=10^{-2} \mathrm{M}$.

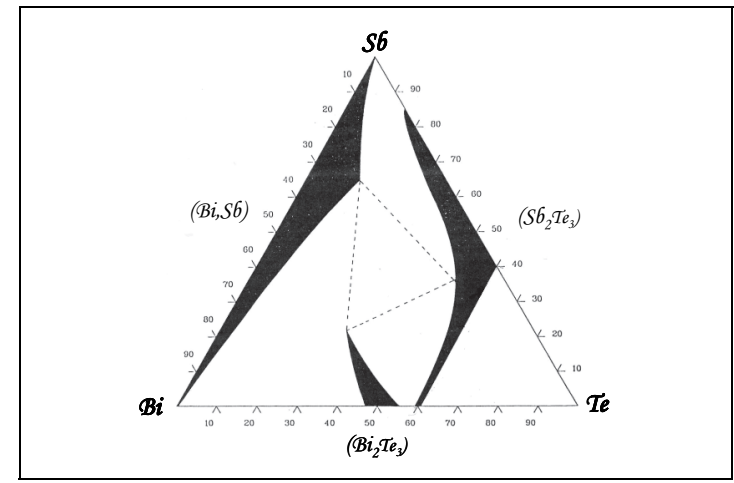

Figure 10. Diagramme de phase du ternaire $\mathrm{Bi}-\mathrm{Sb}-\mathrm{Te}$ $\left(\mathrm{T}=20^{\circ} \mathrm{C}\right)-[7]$.

\subsubsection{Influence du rapport [Sb]/[Bi] dans la solution}

Le diagramme de phase du ternaire $\mathrm{Bi}-\mathrm{Sb}$ - $\mathrm{Te}$ à $\mathrm{T}=20^{\circ} \mathrm{C}$ (Figure 10) indique l'existence d'une solution solide entre $\mathrm{Bi}_{2} \mathrm{Te}_{3}$ et $\mathrm{Sb}_{2} \mathrm{Te}_{3}$. Dans le but d'obtenir toutes les stoechiométries possibles entre ces deux binaires, différents rapports $[\mathrm{Sb}] /[\mathrm{Bi}]$ ont été étudiés $([\mathrm{Sb}] /[\mathrm{Bi}]=2,3,4$ et 6$)$, tout en gardant $([\mathrm{Bi}]+[\mathrm{Sb}]) /[\mathrm{Te}]=1$.

Une étude structurale menée par diffraction des rayons $\mathrm{X}$ sur des dépôts obtenus pour un potentiel de $-170 \mathrm{mV} / \mathrm{ECS}$ est présentée sur la Figure 11. Les diffractogrammes obtenus présentent une meilleure cristallinité quand le rapport [Sb]/[Bi] est petit, c'est-à-dire lorsque le pourcentage d'antimoine est le plus faible, ce qui confirme l'étude cristallographique menée pour le binaire Sb-Te (Figure 6). En outre, on peut noter un léger décalage des pics vers la gauche quand le pourcentage de bismuth dans la solution augmente.

Les résultats stoechiométriques des dépôts effectués au potentiel de $-170 \mathrm{mV} / \mathrm{ECS}$ nous permettent d'étudier la variation de la composition du film en fonction de celle de la solution initiale. Tous les dépôts sont de type $\left(\mathrm{Bi}_{\mathrm{x}} \mathrm{Sb}_{1-\mathrm{x}}\right)_{2} \mathrm{Te}_{3}$, seuls les pourcentages de bismuth et d'antimoine dans le film évoluent en fonction du rapport $[\mathrm{Sb}] /[\mathrm{Bi}]$ dans la solution. Ainsi, on peut noter sur la Figure 12 une parfaite linéarité du pourcentage de bismuth dans le film en fonction de celui de la solution. Au vu de l'équation obtenue, il semble nécessaire d'avoir un pourcentage de 11,6\% de bismuth dans la solution pour atteindre celui de $10 \%$ dans le solide, correspondant à la composition du ternaire $\mathrm{Bi}_{0,5} \mathrm{Sb}_{1,5} \mathrm{Te}_{3}$. Il est donc possible d'obtenir toutes les stoechiométries de dépôt possibles en fonction du rapport $[\mathrm{Sb}] /[\mathrm{Bi}]$ initial de la solution. 


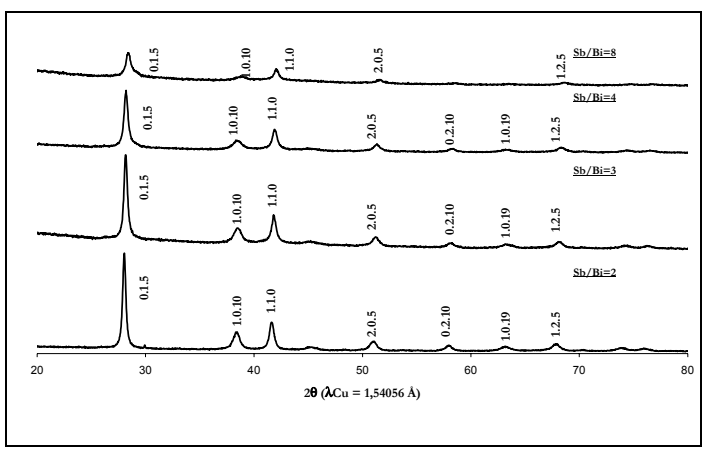

Figure 11. Diffraction des rayons $X$ - Système Bi-Sb-Te - $[\mathrm{Te}]=10^{-2} \mathrm{M} \quad-$ Rapports $[\mathrm{Sb}] /[\mathrm{Bi}]-$ $\mathrm{E}_{\text {déposition }}=-170 \mathrm{mV} / \mathrm{ECS}$.

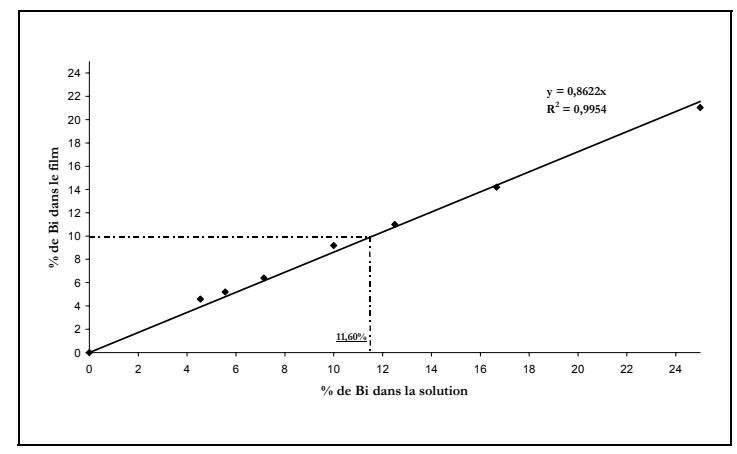

Figure 12. Influence du pourcentage de bismuth dans le film en fonction de celui dans la solution $\mathrm{E}=-170 \mathrm{mV} / \mathrm{ECS}$.

\section{CONCLUSION}

Cette étude nous a permis de connaître le comportement électrochimique des solutions de binaires $\mathrm{Bi}-\mathrm{Te}$ et $\mathrm{Sb}-\mathrm{Te}$, et de ternaire Bi-Sb-Te. De plus, nous avons obtenu, par voie potentiostatique, des dépôts de tellurure de bismuth et d'antimoine $\mathrm{Bi}_{2} \mathrm{Te}_{3}$ et $\mathrm{Sb}_{2} \mathrm{Te}_{3}$, ainsi que des dépôts du ternaire $\mathrm{Bi}_{0,5} \mathrm{Sb}_{1,5} \mathrm{Te}_{3}$ dans une gamme de potentiel de -150 à $-300 \mathrm{mV} / \mathrm{ECS}$. En outre, l'influence de la composition de la solution sur celle du film a été mise en évidence.

L'objectif actuel est d'obtenir ces mêmes résultats par voie galvanostatique, et de déterminer les propriétés physiques de ces films.

\section{Références}

[1] G.S. Nolas, J. Sharp, H.J. Goldsmid, Materials Science, Springer, 2001, "Thermoelectrics: Basics Principles and New Materials Developments"

[2] P. Magri, C. Boulanger, J.M. Lecuire, J. Mater. Chem. 6 (1996) 773

[3] Y. Miyazaki, T. Kajitani, J. Crystal Growth 229 (2001) 542

[4] J.P. Fleurial, A. Borshcheevsky, M.A. Ryan, W.M. Philips, J.G. Snyder, T. Caillat, E.A. Kolawa, J.A. Herman, P. Mueller, M. Nicolet, Mater. Res. Soc. 545 (1999) 493

[5] S. Michel, N. Stein, M. Schneider, C. Boulanger, J.M. Lecuire, J. Appl. Electrochem. 33(1) (2003) 23

[6] S. Michel, Thèse de l'Université de Metz (2003)

[7] P. Villars, A. Prince, H. Okamoto, "Handbook of ternary alloy phase diagrams", Materials Park, OH ASM International (1995) 